\title{
Interoperability Standards for Network Based Airborne Video Systems
}

\author{
Mark Buckley
}

Telspan Data, Concord CA USA

mbuckley@telspandata.com

\begin{abstract}
:
The deployment of network-based airborne instrumentation is leading to cost efficient replacement of legacy systems. One application of airborne instrumentation systems that has to this point been developed and maintained separately from traditional avionics and orange-wire data acquisition systems is video and imagery. The development of network-based video and imagery systems has led to an opportunity to unify these two previously distinct airborne data acquisition activities using standards for plug-n-play interoperability across airframes and organizations. This paper describes standards based network-based video and imagery instrumentation systems which, are being implemented to replace existing proprietary systems.
\end{abstract}

Key words: Instrumentation, Cameras, Network, Video, Imagery, Standards.

\section{Background}

In the late 90's the Central Test and Evaluation Investment Program (CTEIP) funded the Airborne Separation Video System (ASVS) whose goal was to utilize digital imaging cameras as a replacement for existing 400 frames per second (fps) film cameras in the airborne environment. Although there were many different Major Range Test Base Facilities (MRTFB) requirements, a specification was written and funding allocated for the development and procurement of the ASVS system. The goal of this CTEIP task was to create a de-facto standard for digital image collection, storage, and evaluation by developing and procuring a single vendor designed system.

ASVS was comprised of electronicallyshuttered, high-speed, high-resolution digital imaging cameras, a high-density digital data storage device and associated electronics. Although the ASVS program yielded components that are in use today, the hardware systems were built to a specification that was proprietary to the manufacturer. These systems cannot be replaced with commercial equivalent products since the interfaces, command and control are proprietary. Even if the specification and intellectual property was made available, another outdated de-facto standard would be created with outdated technology.

A more telling limitation with ASVS was the lack of utilizing standards leading to a proprietary solution and barrier to entry for camera and ground station manufacturers. The existing ASVS is disk based and its data format, command and control does not adhere to any known standard.

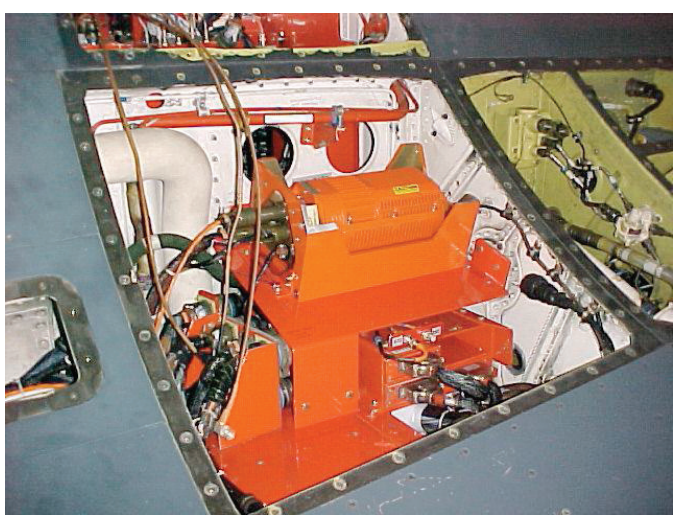




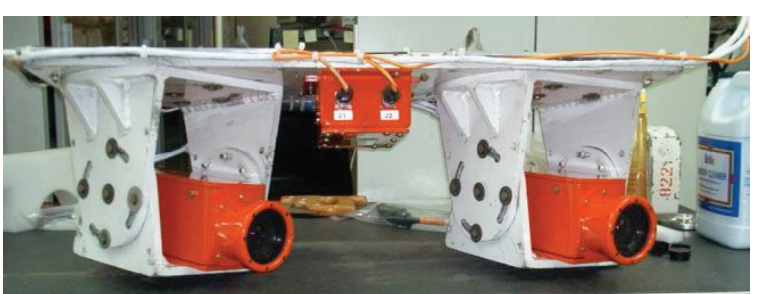

Fig. 1. Legacy ASVS Components and Modification

\section{A New Approach To An Old Requirement}

Using experience gained from the legacy ASVS, a new system is being implemented which is based on industry and Range Commanders Council (RCC) Standards. The key objective of this approach is to implement component and data interoperability for Networked Airborne Video Systems (NAVS) driven by open standards. For example, one requirement is that cameras from different manufacturers be utilized in an on-board Ethernet network with implementation of the existing GigE Vision® (GEV) and IRIG-106 Chapter 10 Digital Recording Standards. The infrastructure of this video network would be based on existing airborne hardware that is already in use in various programs. This new approach would also leverage existing technologies for a generic non-intrusive instrumentation package. The goal is to adopt a system such that it can provide networkbased cameras and that can be backwards compatible (in form and fit) with the existing legacy ASVS.

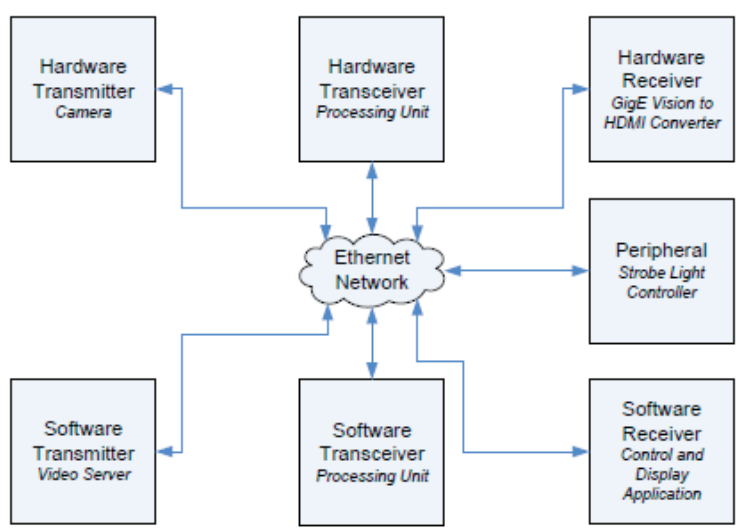

Fig. 2. GigE Vision® Architecture

Existing ASVS systems are being replaced with cameras and other devices compliant with a new RCC Optical Systems Group (OSG) Airborne Network Camera Standard (ANCS) for NAVS implementations. The ANCS is comprised of the following sections:

- System Devices and Operation Requirements; which define the types of on-board cameras and capabilities within the OSG standard.

- GEV Operational Requirements; bootstrap registers and device discovery.

- GenICam Features; derived from the European Machine Vision Association (EMVA) standard for exposing generic camera and device control attributes. These consist of but are not limited to device control of image format, acquisition, exposure, buffers, local media, events, analog, transport layer, user set, time sources and file access.

- GEV Control Protocol (GVCP); which is a protocol to configure devices, instantiate stream and message channels.

- GEV Streaming Protocol (GVSP); provides a mechanism for GVSP transmitters (i.e. cameras) to send image data, image status and other data to GVSP receivers.

- Camera On-Board Acquisition Storage; for video or imagery In Accordance With (IAW) the IRIG 106 Chapter 10 Digital Recording Standard.

NAVS and the standards that it adheres to establishes and promotes vendor independent plug-n-play implementation of network devices (i.e. controllers, switches, cameras, strobe lights and recorders) in an airborne platform. The same wiring that was utilized for legacy ASVS cameras can be utilized for new Ethernet based NAVS implementations. 


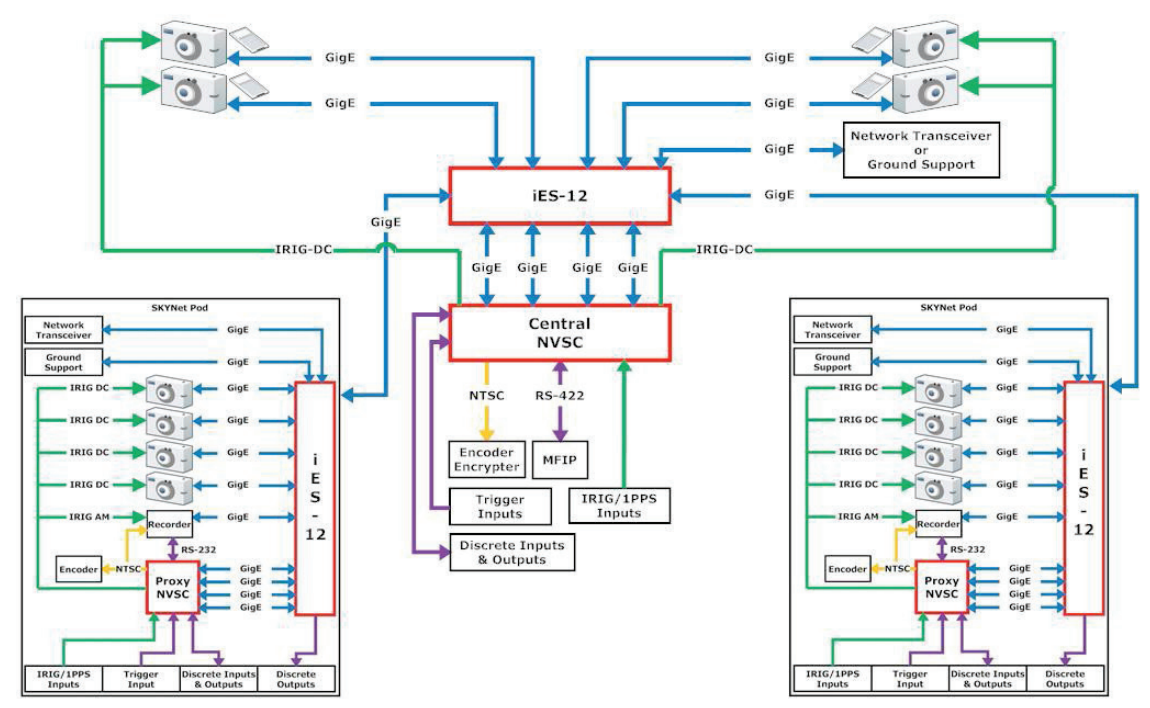

Fig. 3. Example NAVS Topology

Since the same Gigabit Ethernet (GigE) wiring is utilized, the legacy ASVS system can be quickly replaced. Internal to the aircraft, a pallet will be utilized to replace the existing ASVS controller and install NAVS components. The NAVS replacement pallet consists of a systems controller, Ethernet switches, a network IP transceiver, and IRIG-106 Chapter 10 solid state recorder.

The systems controller and Ethernet switches

are utilized to provide IEEE-1588 time to all the

network components and provide command and control from the ground over an 802.11a link. The aircraft interface and camera management is provided by the systems controller which interfaces to the instrumentation control system. The controller also provides NTSC real time video preview from any camera and monitors the weapons release and manual run inputs.

The use of bi-directional connectivity allows real time mission configuration and data analysis, a capability never realized by ASVS. Data from

the cameras is stored IAW IRIG-106 Chapter

10 on either local or remote media storage. Different types of cameras can be utilized in the networked system as long as they are compliant with the RCC OSG ANCS Standard.

\section{Airborne Cameras}

Cameras compliant with the ANCS Standard may operate in stand-alone or as an integral component of NAVS. In NAVS you may have a single or multiple cameras. Strict adherence to the GEV and ANCS Standards is required to facilitate interoperability of cameras from different manufacturers. With "on-board" cameras such as with an aircraft platform, it is critical for NAVS to be automated with minimum operator assistance. In its most basic form, the requirement for an on-board ANCS camera is to trigger, capture the data of interest, and store the captured data to nonvolatile storage.

There are four (4) types of camera architectures defined in the ANCS Standard:

- Live Streaming Only (Type I Camera).

- Live Streaming with Buffered Storage (Type Il Camera).

- Live Streaming with Buffered Storage and Data Acquisition Storage (Type III Camera).

- Live Streaming with Buffered Storage, Data Acquisition Storage and Stored Data Transfer (Type IV Camera).

A Type I camera provides live streaming of images at a programmable rate utilizing SCPO IAW the GEV standard.

A Type II camera provides live streaming of images utilizing at a programmable rate utilizing SCPO IAW the GEV standard with multiple buffers. This type of camera provides multiple sequential acquisitions to multiple memory buffers. Transfer of data from the buffers is accomplished utilizing SCP1 IAW GEV and ANCS Standards.

A Type III camera provides live streaming of images at a programmable rate utilizing SCPO IAW the GEV standard and acquisition data storage to removable memory IAW the ANCS Standard. 
A Type IV camera provides live streaming of images at a programmable rate utilizing SCPO IAW the GEV standard and acquisition data storage to local or removable memory IAW the ANCS Standard.

\section{Network Video Systems Controller (NVSC)}

The NVSC is an airborne GigE Vision receiver and primary control application with triggering, time source and multiplexed video encoder used by NAVS. With multiple Ethernet interfaces the NVSC provides a streaming GigE Vision video imagery receiver to NTSC/H.264 output conversion for pre/post trigger monitoring of cameras in selectable single, dual or quad views. Control of NAVS cameras is accomplished via GVCP with automated setup from internal configuration files or external commands. Camera triggering is provided via external trigger discretes, Mil-Std-1553 bus message monitoring or external commands via UART or Ethernet.

The NVSC can also be configured as a Grand Master time source via a high accuracy internal GPS receiver and hardware based IEEE1588v2 time engine. An internal IRIG Time Code Generator (TCG) is used to drive the IRIG time outputs to cameras which can be seeded from GPS, IEEE-1588v2 or IRIG/1PPS.

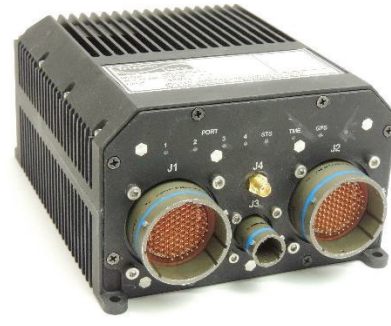

Fig. 5. NAVS Controller

\section{Integrated Ethernet Switches}

iES-6 and iES-12 are airborne 6/12 port layer 2 managed gigabit Ethernet switches with integrated end node timing and discrete signal capabilities used by NAVS. Programmable or data driven discrete outputs can be used to control end node devices on or off the network as well as feedback into the switches from discrete inputs.

The iES-12 also contains a Field Programmable Gate Array (FPGA) tied directly to the switch backplane for user defined Ethernet traffic processing, filtering, logic or data driven discretes. Both the 6 and 12 port switches contain a Central Processing Unit (CPU) for switch management, content aware processing and responding to GEV device discovery protocol.
With multiple time sources and outputs these switches provide end node device time signals. The iES-12 contains a high accuracy internal GPS receiver and both switches contain a hardware based IEEE-1588v2 time engine able to drive an internal IRIG-A/B/G TCG for time outputs. The iES-12 IEEE-1588v2 time engine can be seeded from the GPS time to act as a grand master clock or configured as a boundary clock, ordinary clock or transparent clock. The GigE Vision Standard V2.0 implements and requires IEEE-1588v2 to be a compliant device.

An integrated board stack architecture and front panel allow for NAVS specific functions and connector interfaces to be implemented without compromising system architecture or integrity.
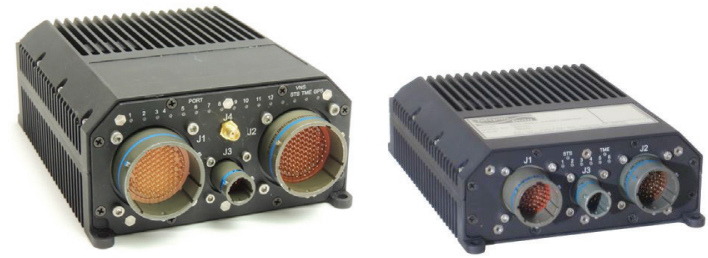

Fig. 6. NAVS Ethernet Switches (12/6 Ports)

\section{Real-Time Full Duplex Command, Control and Data}

The flexibility and capability achieved in creating NAVS is augmented by using IP transceivers to interconnect the airborne instrumentation system with a ground network. These transceivers act as a wireless router for communicating IP packets between NAVS and the ground network infrastructure.

Communicating with the ground station via telnet and the NVSC, individual cameras can be managed, allowing preview of the current image, adjustments to exposure, color, and illumination, and field of view can all be made in real-time during the actual flight plan. Status from NAVS can be accessed using GVCP, and real-time displays used to convey this information to the operators on the ground. Once a triggered event has been captured by the camera system, individual cameras can be queried and frames download to the ground for review and analysis. Events can repeat if feasible, or changes to operating parameters can be made if environmental conditions change.

\section{Ground Support Software}

As with all airborne instrumentation systems NAVS requires ground support software. This software will be operated by technicians, engineers, systems operators, and analysts. Therefore the critical architectural requirements are disciplined implementations, tight 
integration of the main requirements and ease of use by all different levels of operators. The main requirement areas consist of:

- Network Configuration Management

- Pre-Flight Checkout

- In-Flight Command, Control and RealTime Display

- Post Flight Replay and Processing of Recorded Data

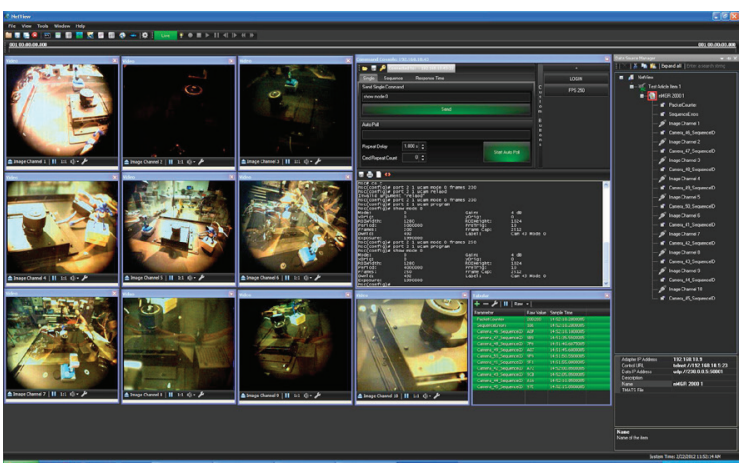

Fig. 7. NAVS Software

As a networked based system all of these software functions can take place on different host computers on the network each having its own function and operator or; just one or two host computers that combine functions under a single operator. The same software components and functions that a pre-flight technician used on the flight line can be used by an engineer during the test to make adjustments to the system. The same software components and functions that a test engineer or analyst uses to view the data can be used by the pre-flight technical during systems setup.

For NAVS totally new software architectures and bottom up development have been implemented. This removes the inefficiencies normally encountered when new approaches and requirements are tacked onto older software programs which end up in layered and disjoint code base. The result is less than desired software execution that causes problems in overall operations for the life of the system. The NAVS program is providing an environment of new innovation in software development such as the NetView Data Fusion application providing combined time aligned processing and display of video, imagery, instrumentation and avionics data.

ANCS simulation and validation software has also been developed which allows engineers to independently implement the requirements of the NAVS systems and standards then perform compliancy testing to ensure interoperability. This removes all in-house and tightly coupled integration efforts which can be costly and time consuming. Systems development and integration can now take place at dispersed geographical locations and manufactures. If the standards have been complied with, the simulation and validation software used then when the NAVS components are integrated onto a test article the result is known operability, performance and ground support.

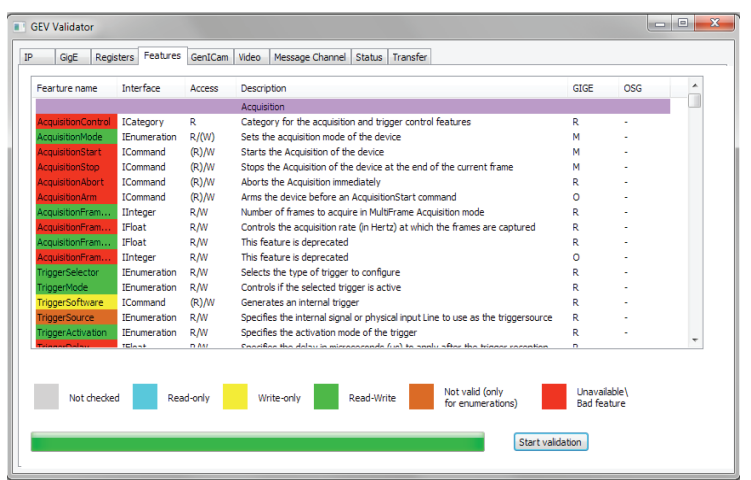

Fig. 8. ANCS Simulation and Validation Software

\section{Conclusion}

By using the ANCS and IRIG 106 Chapter 10 standards along with Ethernet based network technologies NAVS is providing long sought after plug-n-play interoperability in replacing legacy ASVS implementations. Cameras, controllers, network switches and ground software that are compliant with these standards ensure the network, command and control and data systems all work together seamlessly with known performance metrics.

With the ground support software developed for NAVS the concept of data fusion between test data acquisitions systems, platform avionics and on-board video and imagery systems has become a reality. This provides end users the ability analyze data in a manner never attempted before in legacy systems with time aligned data from different systems in single integrated processing and display environment. 